\section{Revisão sistemática sobre morbidade materna near miss}

\author{
Systematic review of near miss \\ maternal morbidity
}

João Paulo Souza ${ }^{1}$
José Guilherme Cecatti ${ }^{1}$
Mary Angela Parpinelli 1
Maria Helena de Sousa ${ }^{1}$
Suzanne Jacob Serruya ${ }^{2}$

\section{Introdução}

This systematic literature review on maternal near miss aims to evaluate data on the incidence and different operational definitions of near miss. An electronic search was performed in databases of scientific journals and also in the references of the identified studies. Initially, 1,247 studies were identified, 35 of which were comprehensively assessed, with 17 excluded and 18 included. Review of reference lists from these articles identified an additional 20 articles, thus completing 38 studies included: 20 adopting definitions of near miss related to management complexity, 6 to organ dysfunction, 2 with a mixed definition, and 10 according to symptoms, signs, or specific clinical entities. The mean near miss ratio was 8.2/1,000 live births, the maternal mortality index was $6.3 \%$, and the case/fatality ratio was 16:1. The study concluded that there was a trend towards higher incidence of near miss in developing countries and when using near miss definitions by organ dysfunction. The study of near miss maternal morbidity can help improve obstetric care and support the struggle against maternal mortality.

Maternal Mortality; Morbidity; Pregnancy Complications
A Organização Mundial da Saúde (OMS) estima que anualmente 20 milhões de mulheres apresentam complicações agudas da gestação, com a ocorrência de 529 mil óbitos 1 . A ocorrência da morte materna extrapola a tragédia individual, constituindo um aspecto de avaliação do desenvolvimento humano ${ }^{2}$. Em países da África Sub-saariana, a morte materna é um evento freqüente, capaz de produzir uma razão de morte materna tão elevada quanto 1.500 óbitos por 100 mil nascidos vivos, enquanto que nos países desenvolvidos as razões de morte materna são baixas, de 10 ou menos óbitos por 100 mil nascidos vivos 1 . No Brasil, o óbito materno é um problema subdimensionado, tendo sido estimado em 2002, cerca de 73 óbitos maternos por 100 mil nascidos vivos 3 .

A dispersão desses óbitos pelo território do país faz com que eles sejam eventos institucionalmente muito raros, o que impede o reconhecimento da mortalidade materna como problema de saúde pública e dificulta o estudo dos determinantes da mortalidade materna. No Brasil, em 2001, nasceram cerca de 3.106.525 nascidos vivos em aproximadamente 5.390 hospitais, com cerca de 1.600 de óbitos maternos notificados 3 . Esses números indicam que, se a distribuição fosse homogênea, ocorreria cerca de 0,3 óbito materno por hospital que atende partos por ano no Brasil. De qualquer forma, 
mesmo em serviços de referência que atendem mulheres com complicações na gravidez, o número absoluto de mortes maternas é pequeno, pois o evento é incomum.

Na última década, especialmente nos países desenvolvidos, os estudos sobre as causas de morte materna começaram a tratar, cada vez mais, de ocorrências muito incomuns, cuja freqüência é praticamente irrelevante para a assistência obstétrica da maioria das mulheres 4 . Contudo, persiste a preocupação com a adequada assistência à mulher, voltada para as que apresentam morbidade grave durante a gravidez, parto ou puerpério, mesmo em países com baixíssimas razões de morte materna, uma vez que para cada óbito materno ocorrido, um considerável número de mulheres desenvolve morbidade grave e apresenta seqüelas permanentes desde então 5,6. Mais do que isso, o adequado enfrentamento de complicações ocorridas no ciclo grávido puerperal tem sido colocado como crucial na estratégia de combate à mortalidade materna 5 .

O interesse em quadros de complicação severa em obstetrícia propiciou a adaptação, para as ciências médicas, de um conceito desenvolvido pela indústria aeronáutica para descrever incidentes de aproximação indevida de aeronaves nas operações de controle de tráfego aéreo. O conceito de "quase-perda", que será mantido nesta revisão na sua forma original em inglês, near miss, referia-se originalmente a um choque de aeronaves durante o vôo que esteve próximo de ocorrer, e que somente não ocorreu por um bom julgamento ou sorte 7 . Do ponto de vista militar, o conceito near miss refere-se ao projétil balístico que erra por pouco seu alvo. No estudo da morbidade materna, o conceito near miss foi introduzido por Stones et al. 8, e faz referência à situação onde mulheres que apresentam complicações potencialmente letais durante a gravidez, parto ou puerpério, somente sobrevivem devido ao acaso ou ao cuidado hospitalar 9 .

Essas mulheres, às quais se pode atribuir o conceito near miss, são de grande interesse para o estudo da morbi-mortalidade materna, uma vez que constituem um grupo muito mais freqüente e com melhor fonte de informação que os casos de morte materna. Também por esta razão, o estudo da near miss materna tem sido utilizado para auditar a qualidade do cuidado obstétrico do ponto de vista hospitalar e como grupo de comparação em estudos de casos de morte materna 6 .

O conceito geral de near miss está estabelecido, mas ainda há controvérsia sobre a sua operacionalização na definição dos casos. Al- guns pesquisadores adotam a ocorrência de disfunção orgânica materna, outros baseiamse em determinadas doenças (por exemplo, eclâmpsia), e outros ainda a partir do grau de complexidade do manejo assistencial (por exemplo, admissão em UTI ou realização de histerectomia) 4,10,11,12,13,14,15,16. Mais recentemente, foi proposta uma abordagem múltipla, baseada em um escore que procura diferenciar a morbidade materna grave daquilo que poderia ser de fato chamado de near miss, por meio do julgamento clínico do especialista, utilizando como indicadores o diagnóstico de doenças, de eventos mórbidos e a utilização de procedimentos 17,18,19.

Considerando a relevância deste tema e a inexistência de um consenso sobre qual definição operacional de near miss deve ser recomendada, os objetivos desta revisão foram analisar as diferentes definições operacionais de near miss utilizadas nos diversos estudos da literatura, a incidência compilada de near miss relatada e outros resultados obtidos.

\section{Método}

Este é um estudo de revisão sistemática realizado por meio de busca eletrônica nos bancos de dados de periódicos científicos MEDLINE e SciELO, utilizando-se os unitermos "near miss maternal mortality”, “near miss maternal morbidity", "near miss obstetric", "severe maternal morbididty" e "severe obstetric morbidity". No MEDLINE, a busca foi realizada com os seguintes limites "human" e "female".

Foi desenvolvido um protocolo para manejar o grande número de estudos inicialmente identificados. Nesse protocolo, todos os estudos cujos títulos ou resumos mencionassem a apresentação de dados originais sobre a morbidade materna near miss ou julgados pertinentes ao tema em estudo foram obtidos na íntegra e a seguir analisados. Este subconjunto de estudos foi avaliado quanto à presença de dados originais e a possibilidade de determinação da incidência de near miss, sendo este o critério de elegibilidade adotado. Os estudos que não apresentaram dados originais ou cujos dados não permitiram a determinação da incidência de near miss, ou que estudaram populações específicas (por exemplo, estudos referentes à incidência de near miss em mulheres hipertensas etc.) foram considerados não elegíveis para a inclusão na presente revisão sistemática.

Todos os estudos analisados na íntegra tiveram suas listas de referências bibliográficas 
revisadas, visando à identificação de outros estudos relacionados à morbidade materna near miss e eventualmente não identificados pela busca eletrônica. Esta estratégia foi aplicada por dois revisores de forma independente e eventuais discordâncias quanto à elegibilidade de determinado estudo foram resolvidas de forma consensual entre os revisores.

A Figura 1 traz um fluxograma que resume a estratégia adotada para identificação e inclusão dos estudos. Os estudos incluídos foram avaliados quanto: período de estudo, desenho, população estudada (número de nascidos vivos e de partos), local, informações sobre sujeitos excluídos e definições operacionais de near miss materna e de entidades clínicas (por exemplo, a definição adotada de pré-eclâmpsia grave), número de mortes maternas e número de near miss. A definição operacional de near miss utilizada em cada estudo foi classificada em uma das quatro seguintes categorias: complexidade de manejo, sinais e sintomas, disfunção orgânica, ou mista 6,20.

Foi determinado o índice de mortalidade materna de cada estudo, conforme descrito por Vandecruys et al. 21, no qual o número de mortes maternas é dividido pela soma das mortes maternas com o número de mulheres que desenvolveram a morbidade aguda grave (near miss). Nesta revisão, apenas médias foram utilizadas para a estimativa dos indicadores compilados dos diferentes estudos incluídos.

\section{Resultados}

A busca eletrônica em base de dados resultou na identificação de 1.247 estudos, sendo que 35 possuíam títulos ou resumos que mencionavam a apresentação de dados originais sobre a morbidade materna near miss ou foram julgados pertinentes ao tema em estudo. Destes, 17 estudos foram excluídos por não apresentarem dados suficientes para determinação da incidência de near miss e 18 foram incluídos. A Tabela 1 identifica, para cada um dos estudos excluídos, o motivo principal da exclusão. A revisão das listas de referências dos 35 artigos analisados na íntegra levou à inclusão de mais vinte estudos, totalizando 38 incluídos (Figura 1).

A grande maioria dos estudos analisados possui o desenho de corte transversal (34), enquanto há apenas três coortes populacionais e um estudo caso-controle. Dezenove estudos foram realizados em países desenvolvidos e igual número nos países em desenvolvimento. Vinte estudos adotaram definições relacionadas à complexidade do manejo; em seis estudos a de- finição estava relacionada à disfunção orgânica; em dez a definição de near miss relacionava-se à presença de determinados sinais e sintomas ou entidades clínicas específicas e, em dois estudos foi adotada uma definição mista de near miss. Nos estudos onde a definição de near miss esteve relacionada à complexidade do manejo, em 19 deles isto significou admissão à UTI, sendo que um deles adotou também o critério da ventilação mecânica 22 , e em outro, a realização de histerectomia foi o indicativo de near miss 23. Em todos os estudos, nos quais a definição de near miss esteve relacionada à disfunção orgânica, foram adotados os critérios propostos por Mantel et al. 16. Na categoria de estudos cujas definições foram baseadas em condições clínicas, houve maior heterogeneidade quanto às definições devido a diferenças nos critérios diagnósticos para as doenças e em decorrência de não serem as mesmas doenças ou condições em cada estudo. Entretanto, todos os estudos trouxeram as seguintes condições como indicativas de possível near miss: desordens hipertensivas, hemorragia grave e sepse (Tabela 2).

A Tabela 3 sumariza as incidências de near miss e de mortalidade materna, apresenta os índices de mortalidade materna e as razões de caso:fatalidade de cada estudo, bem como os valores médios para o total deles e para os subgrupos por diferentes tipos de critérios. Foi identificada tendência de maior mortalidade nos estudos que fizeram uso da definição de near miss relacionada à disfunção orgânica, enquanto uma menor mortalidade esteve associada às definições que relacionam a presença de determinados sinais e sintomas à condição de near miss.

O índice de mortalidade materna e a razão de near miss por mil partos dos países desenvolvidos foram, respectivamente, de 3,0\% e 3,4 casos/mil partos, enquanto que nos países em desenvolvimento estes indicadores foram de $7,5 \%$ e 18 casos/mil partos (dados não apresentados em tabela).

\section{Discussão}

Do ponto de vista conceitual, existe um espectro de gravidade clínica que possui em suas extremidades, de um lado, a gravidez saudável e, do outro, o óbito materno. Neste continuum insere-se um determinado grau de morbidade grave compatível com o conceito near miss. A controvérsia existente sobre uma definição operacional, consensual e generalizável para o termo near miss é conseqüência da dificuldade 
Figura 1

Representação esquemática do método de busca e dos resultados obtidos: estudos excluídos e incluídos.

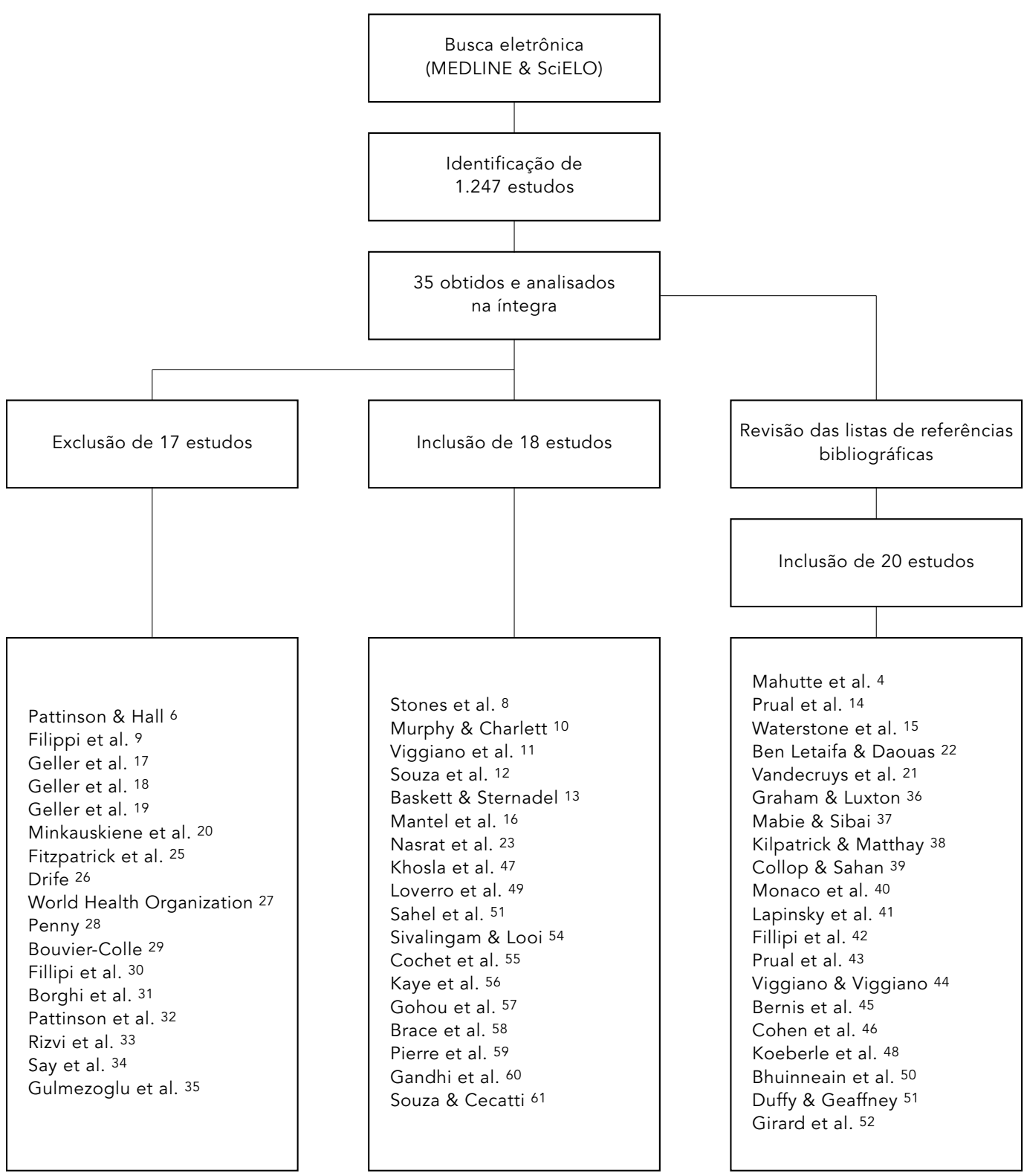

Critério de inclusão: estudo sobre near miss materna com dados originais ou com dados suficientes para a determinação da incidência de near miss.

Critério de exclusão: estudo sobre near miss materna sem dados originais ou com dados insuficientes para a determinação da incidência de near miss. 
Estudos de near miss materna excluídos e critérios de exclusão na revisão da literatura.

\begin{tabular}{|c|c|c|}
\hline Estudo & Data & Motivo da exclusão \\
\hline Fitzpatrick et al. 25 & 1992 & Dados insuficientes para determinação da incidência de near miss \\
\hline Drife 26 & 1993 & Dados insuficientes para determinação da incidência de near miss \\
\hline World Health Organziation 27 & 1994 & Ausência de dados originais sobre a incidência de near miss \\
\hline Penny 28 & 1999 & Ausência de dados originais sobre a incidência de near miss \\
\hline Bouvier-Colle 29 & 1999 & Ausência de dados originais sobre a incidência de near miss \\
\hline Filippi et al. 30 & 2000 & Dados insuficientes para determinação da incidência de near miss \\
\hline Geller et al. 17 & 2002 & Dados insuficientes para determinação da incidência de near miss \\
\hline Borghi et al. 31 & 2003 & Dados insuficientes para determinação da incidência de near miss \\
\hline Pattinson \& Hall 6 & 2003 & Ausência de dados originais sobre a incidência de near miss \\
\hline Pattinson et al. 32 & 2003 & Dados insuficientes para determinação da incidência de near miss \\
\hline Filippi et al. 9 & 2004 & Dados insuficientes para determinação da incidência de near miss \\
\hline Minkauskiene et al. 20 & 2004 & Ausência de dados originais sobre a incidência de near miss \\
\hline Rizvi et al. 33 & 2004 & Dados insuficientes para determinação da incidência de near miss \\
\hline Say et al. 34 & 2004 & Ausência de dados originais sobre a incidência de near miss \\
\hline Gulmezoglu et al. 35 & 2004 & Ausência de dados originais sobre a incidência de near miss \\
\hline Geller et al. 18 & 2004 & Dados insuficientes para determinação da incidência de near miss \\
\hline Geller et al. 19 & 2004 & $\begin{array}{l}\text { A origem e o tempo de coleta dos dados de mortalidade diferem } \\
\text { da origem e tempo de coleta dos dados de morbidade grave }\end{array}$ \\
\hline
\end{tabular}

Características dos estudos incluídos.

\begin{tabular}{|c|c|c|c|c|}
\hline Estudo & Data & Local & Desenho & Definição sintética \\
\hline Graham \& Luxton 36 & 1989 & Grã-Bretanha & Corte transversal & Transferência para UTI \\
\hline Mabie \& Sibai 37 & 1990 & Estados Unidos & Corte transversal & Transferência para UTI \\
\hline Stones et al. 8 & 1991 & Grã-Bretanha & Corte transversal & $\begin{array}{l}\text { Episódios ameaçadores de vida: hemorragia obstétrica com perda } \\
\text { de sangue > } 2 \text { l; doença hipertensiva com rápida deterioração dos sinais } \\
\text { clínicos; embolismo pulmonar; Morbidade maior relacionada à anestesia; } \\
\text { ruptura uterina; CA cervical levando à hemorragia antes do parto. }\end{array}$ \\
\hline Kilpatrick \& Matthay 38 & 1992 & Canadá & Corte transversal & Transferência para UTI \\
\hline Collop \& Sahan 39 & 1993 & Estados Unidos & Corte transversal & Transferência para UTI \\
\hline Monaco et al. 40 & 1993 & Estados Unidos & Corte transversal & Transferência para UTI \\
\hline Lapinsky et al. 41 & 1997 & Canadá & Corte transversal & Transferência para UTI \\
\hline Baskett \& Sternadel 13 & 1998 & Canadá & Corte transversal & Transferência para UTI \\
\hline Filippi et al. 42 & 1998 & Benin & Corte transversal & $\begin{array}{l}\text { Eclâmpsia, hemorragia, infecção puerperal, gravidez ectópica, trauma } \\
\text { uterino associado ao aborto, complicações anestésicas. }\end{array}$ \\
\hline Mantel et al. 16 & 1998 & África do Sul & Corte transversal & $\begin{array}{l}\text { Disfunção aguda de órgão e sistemas orgânicos: cardíaca; vascular; } \\
\text { imunológica; respiratória; renal; do fígado; metabólica; de coagulação } \\
\text { e cerebral. } \\
\text { Critérios baseados no manejo: admissão à UTI; histerectomia } \\
\text { de emergência e acidentes anestésicos. }\end{array}$ \\
\hline Prual et al. 43 & 1998 & Nigéria & Corte transversal & $\begin{array}{l}\text { Hemorragia grave, desordens hipertensivas graves, sepse, distócia } \\
\text { grave e outras complicações. }\end{array}$ \\
\hline Mahute et al. 4 & 1999 & Canadá & Corte transversal & Transferência para UTI \\
\hline Nasrat et al. 23 & 1999 & Arábia Saudita & Corte transversal & Necessidade de histerectomia \\
\hline Viggiano \& Viggiano 44 & 2000 & Brasil & Corte transversal & Transferência para UTI \\
\hline Bernis et al. 45 & 2000 & Senegal & Coorte populacional & Complicações clínicas e outras morbidades na gestação. \\
\hline Cohen et al. 46 & 2000 & Israel & Corte transversal & Transferência para UTI \\
\hline
\end{tabular}

(continua) 
Tabela 2 (continuação)

\begin{tabular}{|c|c|c|c|c|}
\hline Estudo & Data & Local & Desenho & Definição sintética \\
\hline Khosla et al. 47 & 2000 & Índia & Corte transversal & Idem à Mantel et al. 16 \\
\hline Koeberle et al. 48 & 2000 & França & Corte transversal & Transferência para UTI \\
\hline Prual et al. 14 & 2000 & $\begin{array}{l}\text { Mauritânia, } \\
\text { Burkina Faso, } \\
\text { Senegal, Mali, } \\
\text { Nigéria e Costa } \\
\text { do Marfim }\end{array}$ & Coorte populacional & $\begin{array}{l}\text { Hemorragia grave, desordens hipertensivas graves, sepse, distócia } \\
\text { grave e outras complicações. }\end{array}$ \\
\hline Loverro et al. 49 & 2000 & Itália & Corte transversal & Transferência para UTI \\
\hline Bhuinneain et al. 50 & 2001 & Irlanda & Corte transversal & Transferência para UTI \\
\hline Duffy \& Geaffney 51 & 2001 & Irlanda & Corte transversal & Transferência para UTI \\
\hline Girard et al. 52 & 2001 & França & Corte transversal & $\begin{array}{l}\text { Hemorragia grave, desordens hipertensivas graves, sepse, distócia } \\
\text { grave e outras complicações. }\end{array}$ \\
\hline Sahel et al. 53 & 2001 & Marrocos & Corte transversal & $\begin{array}{l}\text { Hemorragia grave, desordens hipertensivas graves, sepse, distócia } \\
\text { grave e outras complicações. }\end{array}$ \\
\hline Waterstone et al. 15 & 2001 & Grã-Bretanha & Caso-controle & $\begin{array}{l}\text { Pré-eclâmpsia, eclâmpsia, síndrome HELLP, hemorragia grave, sepse } \\
\text { grave e ruptura uterina. }\end{array}$ \\
\hline $\begin{array}{l}\text { Ben Letaifa } \\
\& \text { Daouas } 21\end{array}$ & 2002 & Tunísia & Corte transversal & Transferência para UTI e ventilação mecânica \\
\hline Murphy \& Charlett 10 & 2002 & Grã-Bretanha & Corte transversal & Transferência para UTI \\
\hline Sivalingam \& Looi 54 & 2002 & Malásia & Corte transversal & Transferência para UTI \\
\hline Souza et al. 12 & 2002 & Brasil & Corte transversal & Transferência para UTI \\
\hline Vandecruys et al. 21 & 2002 & África do Sul & Coorte populacional & Idem à Mantel et al. 16 \\
\hline Cochet et al. 55 & 2003 & África do Sul & Corte transversal & Idem à Mantel et al. 16 \\
\hline Kaye et al. 56 & 2003 & Uganda & Corte transversal & Idem à Mantel et al. 16 \\
\hline Viggiano et al. 11* & 2004 & Brasil & Corte transversal & Transferência para UTI \\
\hline Gohou et al. 57 & 2004 & Costa do Marfim & Corte transversal & $\begin{array}{l}\text { Distócia grave, doença hipertensiva grave, hemorragia grave, sepse, } \\
\text { anemia grave. }\end{array}$ \\
\hline Brace et al. 58 & 2004 & Escócia & Corte transversal & $\begin{array}{l}\text { Hemorragia grave, eclâmpsia, insuficiência renal, parada cardíaca, } \\
\text { edema pulmonar, insuficiência respiratória, coma, acidente vascular } \\
\text { cerebral, status epilepticus, choque anafilático, choque séptico, } \\
\text { acidente anestésico, admissão à UTI. }\end{array}$ \\
\hline Pierre et al. 59 & 2004 & Escócia & Corte transversal & $\begin{array}{l}\text { Hemorragia grave, complicações da pré-eclâmpsia, choque séptico } \\
\text { e outras complicações. }\end{array}$ \\
\hline Gandhi et al. 60 & 2004 & África do Sul & Corte transversal & $\begin{array}{l}\text { "uma mulher que, em qualquer momento da gestação e até seis } \\
\text { semanas de puerpério, tenha sofrido uma disfunção orgânica aguda } \\
\text { grave, que poderia ter resultado em morte materna caso não tivesse } \\
\text { recebido um tratamento médico adequado"; nos estabelecimentos } \\
\text { secundários e terciários esta definição efetivou-se através dos critérios } \\
\text { propostos por Mantel et al. } 16\end{array}$ \\
\hline Souza \& Cecatti 61 & 2005 & Brasil & Corte transversal & Critérios utilizados por Mantel et al. 16 e Waterstone et al. 15 \\
\hline
\end{tabular}

* Expansão da casuística do estudo anterior do mesmo autor.

para a transformação de uma variável contínua em discreta, uma vez que não é conhecido o melhor ponto de clivagem do espectro de gravidade para a sua categorização 17 .

Nesta revisão, observou-se que dos dezesseis estudos realizados em países desenvolvidos, treze utilizaram as definições operacionais de near miss baseadas na complexidade do manejo, enquanto que, em países em desenvolvimento, seis estudos foram realizados com essa definição operacional. Constatou-se a tendência, na amostra de estudos avaliada nesta revisão, dos pesquisadores de países desenvolvidos utilizarem uma definição operacional de near miss baseada na complexidade do manejo. Entretanto, a organização de cada serviço, como também a disponibilidade de leitos de terapia intensiva, podem representar um considerável viés, uma vez que serviços que dispõem de leitos mais facilmente tendem a realizar a indicação de terapia intensiva de forma mais precoce e às vezes até preventiva, enquanto que serviços com maior limitação de leitos tendem a admitir as mulheres em estado mais grave, muitas vezes quando o processo letal já se encontra deflagrado, resultando em maior mortalidade.

As definições baseadas em condições clínicas estão, em geral, centradas nas maiores cau- 
Classificação dos estudos, segundo diferentes critérios de near miss.

\begin{tabular}{|c|c|c|c|c|c|c|c|}
\hline Estudo & Data & $\mathrm{n}$ & $\begin{array}{c}\text { Near miss + } \\
\text { óbitos maternos }\end{array}$ & $\begin{array}{l}\text { Óbitos } \\
\text { maternos }\end{array}$ & $\begin{array}{c}\text { Near miss/ } \\
1.000\end{array}$ & $\begin{array}{l}\text { Índice de morte } \\
\text { materna }(\%)\end{array}$ & $\begin{array}{c}\text { Caso: } \\
\text { fatalidade }\end{array}$ \\
\hline \multicolumn{8}{|l|}{ Complexidade de manejo } \\
\hline Graham \& Luxton 36 & 1989 & 23.000 & 23 & 2 & 0,9 & 8,7 & $11: 1$ \\
\hline Mabie \& Sibai 37 & 1990 & 22.220 & 200 & 9 & 8,6 & 4,5 & $21: 1$ \\
\hline Kilpatrick \& Matthay 38 & 1992 & 8.000 & 32 & 4 & 3,5 & 12,5 & $7: 1$ \\
\hline Collop \& Sahan 39 & 1993 & 6.667 & 20 & 4 & 2,4 & 20,0 & $4: 1$ \\
\hline Monaco et al. 40 & 1993 & 15.200 & 38 & 7 & 2,0 & 18,4 & $4: 1$ \\
\hline Lapinsky et al. 41 & 1997 & 25.000 & 65 & 0 & 2,6 & - & - \\
\hline Baskett \& Sternadel 13 & 1998 & 76.119 & 55 & 2 & 0,7 & 3,6 & $27: 1$ \\
\hline Mahute et al. 4 & 1999 & 44.340 & 131 & 3 & 2,9 & 2,3 & $43: 1$ \\
\hline Nasrat et al. 23 & 1999 & 18.842 & 23 & 1 & 1,2 & 4,3 & $22: 1$ \\
\hline Cohen et al. 46 & 2000 & 9.200 & 46 & 1 & 4,9 & 2,2 & $45: 1$ \\
\hline Koeberle et al. 48 & 2000 & 1.055 & 46 & 2 & 41,7 & 4,3 & $22: 1$ \\
\hline Bhuinneain et al. 50 & 2001 & 67.650 & 26 & 4 & 0,3 & 15,4 & $6: 1$ \\
\hline Duffy \& Geaffney 51 & 2001 & 20.800 & 19 & 1 & 0,9 & 5,3 & $18: 1$ \\
\hline Ben Letaifa \& Daouas 22 & 2002 & 24.812 & 20 & 6 & 0,6 & 30,0 & $2: 1$ \\
\hline Murphy \& Charlett 10 & 2002 & 51.756 & 50 & 3 & 0,9 & 6,0 & $16: 1$ \\
\hline Sivalingam \& Looi 54 & 2002 & 9.932 & 122 & 10 & 11,3 & 8,2 & $11: 1$ \\
\hline Souza et al. 12 & 2002 & 28.660 & 68 & 23 & 1,6 & 33,8 & $2: 1$ \\
\hline Viggiano et al. 11* & 2004 & 7.936 & 86 & 21 & 8,2 & 24,4 & $3: 1$ \\
\hline Subtotal & & 484.883 & 1.111 & 105 & 2,1 & 9,5 & $10: 1$ \\
\hline \multicolumn{8}{|l|}{ Disfunção orgânica } \\
\hline Mantel et al. 16 & 1998 & 13.854 & 147 & 30 & 8,4 & 20,4 & $4: 1$ \\
\hline Khosla et al. 47 & 2000 & 5.124 & 224 & 31 & 37,7 & 13,8 & $6: 1$ \\
\hline Vandecruys et al. 21 & 2002 & 40.006 & 426 & 85 & 8,5 & 19,9 & $4: 1$ \\
\hline Gandhi et al. 60 & 2004 & 5.728 & 31 & 0 & 5,4 & - & - \\
\hline Subtotal & & 95.535 & 1.177 & 197 & 10,2 & 16,7 & $6: 1$ \\
\hline \multicolumn{8}{|l|}{ Sinais e sintomas } \\
\hline Stones et al. 8 & 1991 & 2.180 & 19 & 0 & 8,7 & - & - \\
\hline Filippi et al. 42 & 1998 & 4.291 & 353 & 30 & 75,3 & 8,5 & $11: 1$ \\
\hline Prual et al. 43 & 1998 & 4.081 & 232 & 21 & 51,7 & 9,0 & $10: 1$ \\
\hline Bernis et al. 45 & 2000 & 3.741 & 277 & 16 & 69,8 & 5,8 & $16: 1$ \\
\hline Prual et al. 14 & 2000 & 20.326 & 1.307 & 41 & 62,3 & 3,1 & $31: 1$ \\
\hline Girard et al. 52 & 2001 & 27.875 & 224 & 1 & 8,0 & 0,4 & $223: 1$ \\
\hline Sahel et al. 53 & 2001 & 5.686 & 81 & 5 & 13,4 & 6,2 & $15: 1$ \\
\hline Waterstone et al. 15 & 2001 & 48.865 & 588 & 5 & 11,9 & 0,8 & $117: 1$ \\
\hline Gohou et al. 57 & 2004 & 14.000 & 1.051 & - & 75,1 & - & - \\
\hline Pierre et al. 59 & 2004 & 15.281 & 50 & 1 & 3,2 & 2,0 & $49: 1$ \\
\hline Subtotal & & 146.326 & 4.182 & 120 & 27,8 & 2,9 & $35: 1$ \\
\hline \multicolumn{8}{|l|}{ Definição mista } \\
\hline Brace et al. 58 & 2004 & 51.165 & 200 & 4 & 3,83 & 2,0 & $49: 1$ \\
\hline Souza \& Cecatti 61 & 2005 & 2.929 & 126 & 2 & 42,3 & 1,6 & $62: 1$ \\
\hline Subtotal & & 54.094 & 326 & 6 & 5,9 & 1,8 & $54: 1$ \\
\hline Total & & 780.838 & 6.796 & 428 & 8,2 & 6,3 & $16: 1$ \\
\hline
\end{tabular}

* Contém a casuística do estudo anterior do mesmo autor. 
sas de morte materna: hipertensão, hemorragia e sepse 5 . Entretanto, foram observadas diferenças nas definições de cada situação clínica, como, por exemplo, a quantidade de sangue perdido para que a hemorragia seja considerada grave. Uma outra consideração que deve ser feita é a capacidade de enfrentamento de determinada condição e o impacto que esta capacidade tem na mortalidade. Por exemplo, se a perda de cerca de $2.000 \mathrm{ml}$ de sangue for considerada indicativa de near miss, a gravidade associada a esta perda irá variar mediante a capacidade de intervir na hemorragia, como a disponibilidade de sangue ou de um centro cirúrgico. À proporção que trata de eventos clínicos isolados, que não consideram a resposta orgânica da mulher e do meio, ocorre a tendência de incluir casos mais leves dentre os casos near miss. Na presente revisão, de fato essa tendência foi observada, com um índice médio de mortalidade materna para estes estudos utilizando sinais e sintomas como critérios de near miss, ao redor da metade da média do total de estudos. Provavelmente existe uma tendência onde os casos mais graves apresentam maior índice de mortalidade, sendo o contrário também válido. Porém, pretensos indicadores de qualidade como o índice de mortalidade materna só são válidos se for possível realizar um pareamento por gravidade clínica 24 .

Uma terceira abordagem define near miss com base na disfunção orgânica. Do ponto de vista fisiopatológico, essa abordagem é a mais precisa, à medida que considera a resposta orgânica da mulher, independentemente da injúria clínica inicial. Ou seja, a disfunção orgâ- nica reflete a proximidade da morte e, com isso, permite a formação de um conjunto mais uniforme de casos de near miss. Embora seja pequeno o número de estudos avaliados com esta definição, observa-se uma razão caso:fatalidade mais estável, em torno de quatro a seis casos para cada morte. Entretanto, a definição de disfunção orgânica proposta por Mantel et al. 16, e utilizada por outros pesquisadores, apresenta algumas dificuldades para sua operacionalização, à proporção que demanda maior disponibilidade tecnológica e também inclui aspectos da categoria de complexidade de manejo, como a inclusão de mulheres que tenham sido submetidas a histerectomias ou internações em UTI.

Deste modo, ainda inexiste uma definição operacional ideal e o desenvolvimento desta definição demandará a realização de estudos populacionais prospectivos, a serem posteriormente validados em diversas regiões do globo. Contudo, apesar da inexistência de um conceito de near miss materna consensual e generalizável, é necessário que os diferentes serviços adotem uma definição operacional factível e estável ao longo do tempo, considerando a realidade nas quais estão inseridos. Assim, a incidência de near miss varia de acordo com a definição adotada e o grau de desenvolvimento, sendo maior quando se utiliza o critério de disfunção orgânica e nos países em desenvolvimento. A utilização do conceito near miss aplicado à saúde materna pode constituir uma importante ferramenta no combate à morte materna e na melhora da qualidade do cuidado obstétrico oferecido.

\section{Resumo}

Esta revisão sistemática sobre near miss materna objetivou analisar dados de incidência e as definições adotadas de near miss. Procedeu-se uma busca eletrônica em bancos de periódicos científicos e também das referências bibliográficas dos estudos identificados. Foram identificados inicialmente 1.247 estudos, analisados na íntegra 35, sendo 17 excluídos e 18 incluídos. A revisão da lista de referências destes artigos identificou mais vinte, totalizando assim 38 estudos incluídos: vinte com definições de near miss relacionadas à complexidade do manejo, seis de disfunção orgânica, dois com definição mista e dez pela presença de sinais ou entidades clínicas específicas. A razão de near miss média foi de 8,2/mil partos, o índice de mortalidade materna foi 6,3\% e a razão caso:fatalidade de 16:1. Conclui-se que a incidência de near miss tende a ser maior nos países em desenvolvimento e quando utilizada a definição de disfunção orgânica. O estudo da morbidade materna near miss pode contribuir para a melhora da atenção obstétrica e subsidiar o combate à morte materna.

Mortalidade Materna; Morbidade; Complicações na Gravidez 


\section{Colaboradores}

J. P. Souza e J. G. Cecatti participaram de todas as etapas do estudo, incluindo o desenvolvimento do projeto, sendo responsáveis pela coleta e análise dos dados e pela redação do artigo. J. G. Cecatti e M. A. Parpinelli desenvolveram a idéia do projeto, orientaram a implementação do estudo, coleta e análise dos dados e a redação do artigo. M. H. Sousa colaborou na análise, discussão dos resultados e revisão do artigo. S. J. Serruya colaborou na discussão dos resultados e revisão final do artigo.

\section{Referências}

1. World Health Organization. Maternal mortality in 2000: estimates developed by WHO, UNICEF, and UNFPA. Geneva: World Health Organization; 2003.

2. World Health Organization. Reduction of maternal mortality: a joint WHO/UNFPA/UNICEF World Bank Statement. Geneva: World Health Organization; 1999.

3. Departamento de Informática do SUS. Indicadores e dados básicos - Brasil 2004. http://www. datasus.gov.br (acessado em 02/Dez/2005).

4. Mahutte NG, Murphy-Kaulbeck L, Le Q, Solomon J, Benjamin A, Boyd ME. Obstetric admissions to the intensive care unit. Obstet Gynecol 1999; 94:263-6.

5. United Nations Population Fund. Maternal mortality update 2002 - a focus on emergency obstetric care. New York: United Nations Population Fund; 2003.

6. Pattinson RC, Hall M. Near misses: a useful adjunct to maternal death enquiries. Br Med Bull 2003; 67:231-43.

7. Nashef SAM. What is a near miss? Lancet 2003; 361:180-1.

8. Stones W, Lim W, Al-Azzawi F, Kelly M. An investigation of maternal morbidity with identification of life-threatening 'near miss' episodes. Health Trends 1991; 23:13-5.

9. Filippi V, Brugha R, Browne E, Gohou V, Bacci A, De Browere V, et al. Obstetric audit in resource poor settings: lessons from a multi-country project auditing 'near miss' obstetrical emergencies. Health Policy Plann 2004; 19:57-66.

10. Murphy J, Charlett P. Cohort study of near miss maternal mortality and subsequent reproductive outcome. Eur J Obstet Gynecol Reprod Biol 2002; 102:173-8.

11. Viggiano MB, Viggiano MGC, Souza E, Camano L. Necessidade de cuidados intensivos em maternidade pública terciária. Rev Bras Ginecol Obstet 2004; 26:317-23.

12. Souza JPD, Duarte G, Basile Filho A. Near miss maternal mortality in developing countries. Eur J Obstet Gynecol Reprod Biol 2002; 104:80.

13. Baskett TF, Sternadel J. Maternal intensive care and near miss mortality in obstetrics. Br J Obstet Gynaecol 1998; 105:981-4.

14. Prual A, Bouvier-Colle MH, De Bernis L, Bréart G. Severe maternal morbidity from direct obstetric causes in West Africa: incidence and case fatality rates. Bull World Health Organ 2000; 78:593-602.
15. Waterstone $M$, Bewley S, Wolfe C. Incidence and predictors of severe obstetric morbidity: casecontrol study. BMJ 2001; 322:1089-94.

16. Mantel GD, Buchmann E, Rees H, Pattinson RC. Severe acute maternal morbidity: a pilot study of a definition for near miss. Br J Obstet Gynaecol 1998; 105:985-90.

17. Geller SE, Rosenberg D, Cox SM, Kilpatrick S. Defining a conceptual framework for near miss maternal morbidity. J Am Med Womens Assoc 2002; 57:135-9.

18. Geller SE, Rosenberg D, Cox SM, Kilpatrick SJ, Brown ML, Simonson L. A scoring system to identify near-miss maternal morbidity. J Clin Epidemiol $2004 ; 57: 716-20$.

19. Geller SE, Rosenberg D, Cox SM, Brown ML, Simonson L, Driscoll CA, et al. The continuum of maternal morbidity and mortality: factors associated with severity. Am J Obstet Gynecol 2004; 191:939-44.

20. Minkauskiene M, Nadisauskiene R, Padaiga Z, Makari S. Systematic review on the incidence and prevalence of severe maternal morbidity. Medicina (Kaunas) 2004; 40:299-309.

21. Vandecruys H, Pattinson RC, MacDonald AP, Mantel GD. Severe acute maternal morbidity and mortality in the Pretoria Academic Complex: changing patterns over 4 years. Eur J Obstet Gynecol Reprod Biol 2002; 102:6-10.

22. Ben Letaifa D, Daouas N. Maternal emergencies requiring controlled ventilation: epidemiology and prognosis. Eur J Obstet Gynecol Reprod Biol 2002; 31:256-60.

23. Nasrat HA, Youssef MH, Marzoogi A, Talab F. "Near miss" obstetric morbidity in an inner city hospital in Saudi Arabia. East Mediterr Health J 1999; 5:717-26.

24. Knaus WA. APACHE 1978-2001: the development of a quality assurance system based on prognosis: milestones and personal reflections. Arch Surg 2002; 137:37-41.

25. Fitzpatrik C, Halligan A, McKenna P, Coughlan BM, Darling MRN, Phelan D. Near miss maternal mortality (NMM). Ir Med J 1992; 85:37.

26. Drife JO. Maternal "near miss" reports? BMJ 1993; 307:1087-8.

27. World Health Organziation. "1370 mothers reported killed during physiological duty. Thousands more seriously injured". Safe Mother 1994, 14:1-2. 
28. Penny J. Severe acute maternal morbidity: a pilot study of a definition of a near- miss. Br J Obstet Gynaecol 1999; 106:397.

29. Bouvier-Colle MH. Maternal intensive care and near miss mortality in obstetrics. Br J Obstet Gynaecol 1999; 106:1234.

30. Filippi V, Ronsmans C, Gandaho T, Graham W, Alihonou E, Santos P. Women's reports of severe (near-miss) obstetric complications in Benin. Stud Fam Plann 2000; 31:309-24.

31. Borghi J, Hanson K, Ekanmian G, Ronsmans C, Brugha R, Browne E, et al. Costs of near miss obstetric complications for women and their families in Benin and Ghana. Health Policy Plann 2003; 18:383-90.

32. Pattinson RC, Buchmann E, Mantel G, Schoon M, Rees H. Can enquiries into severe acute maternal morbidity act as a surrogate for maternal death enquiries? BJOG 2003; 110:889-93.

33. Rizvi F, Mackey R, McKenna P, Geary M. Successful reduction of massive postpartum haemorrage by use of guidelines and staff education. $\mathrm{Br} \mathrm{J} \mathrm{Ob-}$ stet Gynaecol 2004; 111:495-8.

34. Say L, Pattinson RC, Gulmezoglu AM. WHO systematic review of maternal morbidity and mortality; the prevalence of sevre acute maternal morbidity (near miss). Reprod Health 2004; 1:3.

35. Gulmezoglu AM, Say L, Betrán AP, Villar J, Piaggio G. WHO systematic review of maternal mortality and morbidity: methodological issues and challenges. BMC Med Res Methodol 2004; 4:16.

36. Graham SG, Luxton C. The requirement for intensive care support for the pregnant population. Anaesthesia 1989; 44:581-4.

37. Mabie WC, Sibai BM. Treatment in an obstetric intensive care unit. Am J Obstet Gynecol 1990; 162:1-4.

38. Kilpatrick SJ, Matthay MA. Obstetric patients requiring critical care - a five year review. Chest 1992; 101:1407-12.

39. Collop NA, Sahan S. Critical illness in pregnancy an analysis of 20 patients admitted to a medical intensive care unit. Chest 1993; 103:1548-52.

40. Monaco TJ, Spielman FJ, Katz VL. Pregnant patients in the intensive care unit: a descriptive analysis. South Med J 1993; 86:414-7.

41. Lapinsky SE, Kruczynski K, Seaward GR, Farine D, Grossman RF. Critical management of the obstetric patient. Can J Anaesth 1997; 44:325-9.

42. Filippi V, Alihonou E, Mukantaganda S, Graham WJ, Ronsmans C. Near misses: maternal morbidity and mortality. Lancet 1998; 351:145-6.

43. Prual A, Huguet D, Garbin O, Rabe G. Severe obstetric morbidity of the third trimester, delivery and early puerperium in Niamey (Niger). Afr J Reprod Health 1998; 2:10-9.

44. Viggiano MGC, Viggiano MB. A necessidade de cuidados intensivos e o termo "near miss mortality" aplicado à realidade brasileira. Ginecol Obstet Atual 2000; 9:29-32.

45. Bernis L, Dumont A, Bouillin D, Gueye A, Dompnier JP, Bouvier-Colle MH. Maternal Morbidity and mortality in two different populations of Senegal: a prospective study (MOMA survey). BJOG 2000; 107:68-74.
46. Cohen J, Singer P, Kogan A, Hod M, Bar J. Course and outcome of obstetric patients in a general intensive care unit. Acta Obstet Gynecol Scand 2000; 79:846-50.

47. Khosla AH, Dahiya K, Sangwan K. Maternal mortality and 'near miss' in rural north India. Int J Gynaecol Obstet 2000; 68:163-4.

48. Koerberle P, Levy A, Surcin S. Severe obstetric complications necessitating hospitalization and intensive care: a ten years retrospective study. Ann Fr Anesth Reanim 2000; 19:445-51.

49. Loverro G, Pansini V, Greco P, Vimercati A, Parisi AM, Selvaggi L. Indications and outcome for intensive care unit admission during puerperium. Arch Gynecol Obstet 2001; 265:195-8.

50. Bhuinneaain MN, Barry-Kinsella C, Coughlan BM McKenna PF, Bosio P. Critical care admission of obstetric patients. Ir Med J 2001; 94:51-2.

51. Duffy S, Geaffney G. Maternal admissions to ICU? Time to reevaluate. Ir Med J 2001; 94:64-5.

52. Girard F, Burlet G, Bayoumeu F, Bouvier-Colle $\mathrm{MH}$, Boutroy JL. Les complications severes de la grossesse et de l'accouchement: etat des lieux ne Lorraine dans le cadre de l'enquete europeenne. J Gynecol Obstet Biol Reprod 2001; 30:10-7.

53. Sahel A, De Brouwere V, Lardi M, Van Lerberghe W, Ronsmans C, Filippi V. Des catastrophes obstétricales évitées de justesse: les near miss dans les hôpitaux marocains. Cahiers Santé 2001; 11: 229351

54. Sivalingam N, Looi KW. Clinical experience with management of "near miss" cases in obstetrics. Med J Malaysia 1999; 54:496-503.

55. Cochet L, Pattinson RC, MacDonald AP. Severe acute morbidity and maternal death audit - a rapid diagnostic tool for evaluating maternal care. S Afr Med J 2003; 93:700-2.

56. Kaye D, Mirembe F, Azziga F, Namulema B. Maternal mortality and associated near misses among emergency intrapartum obstetric referrals in $\mathrm{Mu}$ lago Hospital, Kampala, Uganda. East Afr Med J 2003; 80:144-9.

57. Gohou V, Ronsmans C, Kacou L, Yao K, Bohoussou KM, Houphouet B, et al. Responsiveness to lifethreatening obstetric emergencies in two hospitals in Abidjan, Côte d'Ivoire. Trop Med Int Health 2004; 9:406-15.

58. Brace V, Penney G, Hall M. Quantifying severe maternal morbidity: a Scottish population study. BJOG 2004; 111:481-4.

59. Pierre F, Laviso E; Boisselier P, Debaene B, Pourrat O, Magnin G. Quantifying severe maternal morbidity: a Scottish population study. BJOG 2005; 111:259.

60. Gandhi MN, Welz T, Ronsmans C. Severe acute maternal morbidity in rural South Africa. Int J Gynecol Obstet 2004; 87:180-7.

61. Souza JPD, Cecatti JG. The near miss maternal morbidity scoring system was tested in a clinical setting in Brazil. J Clin Epidemiol 2005; 58:962.

Recebido em 14/Out/2004

Versão final reapresentada em 09/Ago/2005

Aprovado em 12/Ago/2005 